\title{
Propuesta de un modelo de medición del capital intelectual que permita diagnosticar su influencia en la competitividad de las empresas agro-exportadoras de Tacna
}

Proposal for a measurement model of intellectual capital that enables diagnose their influence on the competitiveness of the agro-export enterprises of Tacna

\section{Marizol Candelaria Arámbulo Ayala ${ }^{1}$}

\section{RESUMEN}

Objetivo: Proponer un modelo de medición del capital intelectual que permita diagnosticar su influencia en la competitividad de las empresas agroexportadoras de Tacna.

Método: Se realizó una revisión teórica de los diversos enfoques acerca del capital intelectual y de la competitividad, que permitió diseñar una metodología basada en los indicadores propuestos para la medición.

Debido a las características de la investigación, el cuestionario se presenta como la herramienta más apropiada para obtener información específica.

Son pocos los trabajos que han estudiado el capital intelectual en su totalidad, de modo que se han seleccionado e integrado indicadores de medición procedentes de diversas fuentes. Se han formulado una serie de preguntas relacionadas con las variables considerando que el número de preguntas fuera idóneo para dicha aplicación.

Se realizaron las funciones típicas del flujo de procesamiento de datos, se aplicó el análisis factorial exploratorio a cada una de las escalas de medición del modelo, con sus respectivas pruebas y medidas estadísticas, cuyo resultado fue la identificación de los factores de cada componente con sus respectivas cargas factoriales. Adicionalmente, se aplicó el análisis de regresión para diagnosticar el comportamiento de la competitividad a partir del capital intelectual.

Resultado: El modelo de medición consta de 24 indicadores de escala para capital intelectual y 9 para competitividad; y se comprueba que el conjunto de activos del capital intelectual ejercen una influencia positiva en la competitividad.

Conclusiones: Los resultados han permitido constatar el valor estratégico del capital intelectual de su conexión con la competitividad.

\section{PALABRA CLAVE}

Activos intangibles, capital intelectual, conocimiento, competitividad, ventaja competitiva, empresas agroexportadoras.

\begin{abstract}
Objective: Propose a model for measuring intellectual capital that enables diagnose their influence on the competitiveness of agribusiness firms-exporters Tacna.

Method: Review of the various approaches about intellectual capital and competitiveness, which enabled a methodology based on the indicators proposed for measurement.

Due to the nature of the research, the questionnaire is presented as the most appropriate tool for specific information.

Few studies have addressed intellectual capital in full, so that is selected and integrated measurement indicators from various sources, we have designed a series of questions related to the variables where as the number of questions it is suitable for that application.

Functions were performed typical data processing flow; we applied exploratory factor analysis to each of the measurement scales of the model, with their respective tests and statistical measures, which resulted in the identification of the factors of each component with its respective factor loadings. Additionally, regression analysis was used to diagnose the behavior of competitiveness from intellectual capital.

Result: The measurement models 24 scale indicators for intellectual capital, and 9 to competitiveness, and it is found that the set of intellectual capital assets have a positive influence on competitiveness.

Conclusions: Results have been allowed confirm in the strategic value of intellectual capital and its connection with competitiveness.
\end{abstract}

\section{KEYWORDS}

Intangible assets, intellectual capital, knowledge, competitiveness, competitive advantage, agro-export businesses.

1. Doctor en Administración, Magister en Administración y Dirección de Empresas, Ingeniero Industrial. Actualmente Labora como Secretario Académico de la Escuela de Postgrado de la Universidad Privada de Tacna. Especializada en Administración, Logística, Dirección de Operaciones, Auditoria y Tics.

E-mail: marizolarambulo@gmail.com 


\section{Introducción}

La empresa de hoy no es la misma que la de ayer, cotidianamente surgen cambios en el mundo que influyen notoriamente en el diario accionar de cada empresa. Por lo tanto, cada uno de los componentes de ésta debe moldearse para ajustarse de forma óptima a estos acontecimientos. Tradicionalmente, se consideraba que la empresa debía estar conformada por los recursos humanos, financieros y materiales; sin embargo, en este momento a pesar de que se continúan integrando las empresas con estos tres elementos, se requiere además, se utilicen el conocimiento, las habilidades y actitudes de las personas que harán posible que los recursos humanos sean proactivos. Es decir tengan iniciativa para que sean capaces de realizar las actividades inherentes a su puesto con independencia, tomando las decisiones necesarias y correctas para el buen funcionamiento de la empresa; y con esto se pueda lograr no sólo el crecimiento económico sino también el desarrollo humano (Sánchez y Olea, 2000, p.42) de los integrantes de las compañías.

Bajo esta perspectiva, se considera al capital intelectual, incluyendo sus componentes, como una herramienta estratégica de competitividad, necesaria para enfrentar los nuevos desafíos del entorno.

En consecuencia, las empresas necesitan mejorar y para lograrlo, la presente investigación tiene como objetivo proponer un modelo de medición del capital intelectual que permita determinar la influencia de éste en la competitividad de las empresas agroexportadoras de Tacna. Partiendo de la hipótesis de que si se desarrolla un modelo de medición del capital intelectual se contribuirá a diagnosticar su influencia positiva en la competitividad de las empresas agroexportadoras de Tacna.

En fechas recientes, algunos investigadores han intentado dar soporte a esta afirmación, sin embargo los trabajos son aún escasos e incompletos, pues en su mayoría se centran en alguno de los componentes del capital intelectual. Por otra parte, el capital intelectual presenta dificultades en cuanto a su medición, debido a su naturaleza intangible, y aunque existen propuestas para su medición, la investigación en este campo se encuentra aún en su etapa inicial.
Adicionalmente, muchas empresas del país han reflejado la inexistencia de algún modelo idóneo que incorpore el conocimiento, las capacidades y habilidades de los recursos humanos con relación a la productividad, sabiendo que para las empresas más competitivas a escala mundial los activos más valiosos y productivos son las personas.

Suplir estas carencias ha sido motivo para plantear la siguiente investigación, proponiendo un estudio más ambicioso del capital intelectual identificando sus componentes y los factores 0 activos intangibles que los constituyen, proponiendo así escalas para su medición y a partir de allí establecer las relaciones causales que los vinculen con la competitividad, para lo cual se presentan las variables que componen este modelo (ver Figura № 1 ).

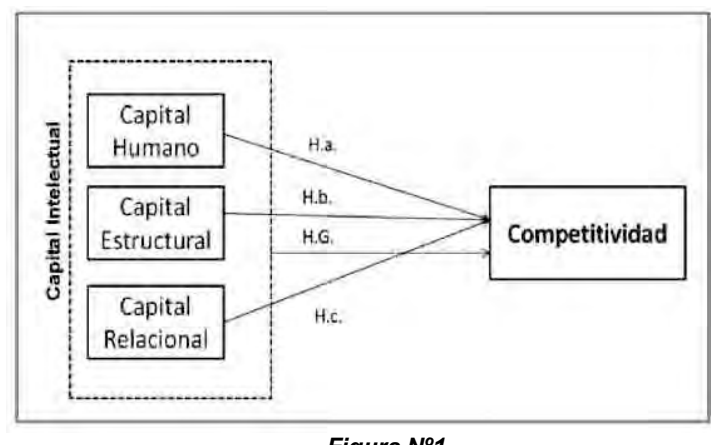

Modelo Específico de la Investigación

\section{Materiales y métodos}

Es una investigación aplicada con presentación cuantitativa de los resultados; no experimental porque explica los fenómenos o situaciones ya existentes en la realidad; $y$ de corte transversal porque la recogida de datos se hizo en un solo momento del tiempo. Es un diseño relacional porque se pretende determinar el nivel de relación que tienen las dos variables de estudio. La elección de la población ha seguido el criterio de seleccionar empresas donde los activos intangibles constituyen los activos más importantes 0 centrales, ya que las competencias distintivas de este tipo de empresas se basan en el conocimiento de los trabajadores, los modos de trabajar, y las relaciones que mantienen con diversos agentes vinculados al negocio, principalmente clientes. Es así que se elige a las empresas agroexportadoras de aceituna específicamente aquellas que conforman la asociación Pro Olivo, que se encuentran debidamente registradas en la base de datos SUNAT. Para la recolección de 
la información se decidió emplear la encuesta como fuente de información primaria elaborando previamente un cuestionario, el mismo que es contestado por una sola persona que se encuentre a un nivel jerárquico superior de cada empresa, pudiendo ser el Gerente General o el Gerente de recursos humanos, considerando el carácter estratégico del Capital Intelectual.

\section{Resultados}

Para que las escalas de medición que se proponen en la investigación sirvan como instrumento de medida en el sector aplicado, es de suma utilidad aplicar el Análisis Factorial Exploratorio (AFE), pues si bien es cierto que se encontraron propuestas para la medición de los componentes del capital intelectual, no se ha determinado cuáles son los factores que los miden. En este sentido, este análisis ha permitido determinar el número de factores relevantes de cada componente del capital intelectual: Capital humano $(\mathrm{CH})$, Capital estructural (CE) y Capital relacional (CR).

Tabla N ${ }^{0} 1$

AFE del Capital Humano

\begin{tabular}{|c|c|c|c|}
\hline \multicolumn{4}{|c|}{ E del Capital Humano } \\
\hline \multirow{2}{*}{\multicolumn{2}{|c|}{ Indicadores de la escala }} & \multicolumn{2}{|c|}{ Factor } \\
\hline & & 1 & 2 \\
\hline \multicolumn{2}{|c|}{ 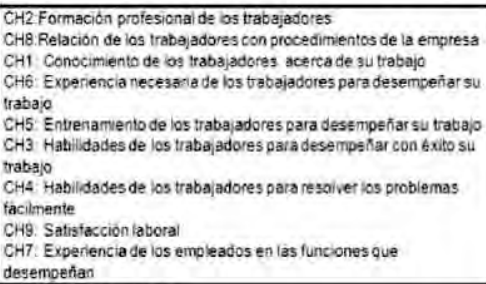 } & $\begin{array}{l}960 \\
879 \\
832 \\
769 \\
749 \\
702\end{array}$ & $\begin{array}{l}618 \\
408\end{array}$ \\
\hline \multicolumn{2}{|l|}{ Şde yarianza expicado } & 46,035 & 20.643 \\
\hline \multicolumn{2}{|l|}{$\%$ de varianza acumulada } & 46.035 & 65.67 \\
\hline \multirow{3}{*}{$\begin{array}{l}\text { Medisa de adecuación m } \\
\text { Prueba de estericidad de } \\
\text { Bartieft }\end{array}$} & straide Kais & \multicolumn{2}{|c|}{638} \\
\hline & Chi-suadrado aprox & \multicolumn{2}{|c|}{112,370} \\
\hline & & \multicolumn{2}{|c|}{$\begin{array}{c}36 \\
000 \\
\end{array}$} \\
\hline
\end{tabular}

El análisis dio a conocer cuántos y cuáles son realmente los factores del capital humano (Tabla $N^{0}$ 1). El primer factor está referido a la formación, habilidades y la relación con el trabajo que desarrollan los trabajadores. Este tiene un porcentaje de varianza explicada de 46,035. El segundo factor está referido a las habilidades para resolver problemas, satisfacción y experiencia adquirida "in situ" es decir, con la práctica y no mediante el conocimiento formal. Este tiene un porcentaje de varianza explicada de 20,643 y que sumadas ambas hacen un total de $66,678 \%$, superando el mínimo exigido del $50 \%$.
Tabla № 2

AFE del Capital Estructural

\begin{tabular}{|c|c|c|}
\hline \multirow{2}{*}{ Indicadores de ta escala } & \multicolumn{2}{|c|}{ Factor } \\
\hline & 1 & 2 \\
\hline $\begin{array}{l}\text { CE3: Politica de innovacion } \\
\text { CEE: Comunicación entre directivos y empleados } \\
\text { CE4: Gasto anuai en Investigaciony desarrolla } \\
\text { CE2: Uso ndinario de sistemas informaticos para reaizar el trabajo }\end{array}$ & $\begin{array}{l}938 \\
890 \\
728 \\
.637\end{array}$ & \\
\hline $\begin{array}{l}\text { CE1. Uso de téécicas informátlcas para realizar el trabajo } \\
\text { CEG: Conocimiento organizativo } \\
\text { CEB Rotación de personal } \\
\text { CES: Desarrollo de programas de calidad }\end{array}$ & & $\begin{array}{l}.863 \\
.825 \\
532 \\
.518\end{array}$ \\
\hline \%de varianza explicada & 35,153 & 26,663 \\
\hline \% de varianza acumulada & 35.153 & 61,816 \\
\hline $\begin{array}{l}\text { Medida de adecuación muestral de Kaiser-Mleyer-Olkin } \\
\text { Prueba de esfericidad de Chi-cuadrado aproximado } \\
\text { Bartett } \\
\qquad \begin{array}{ll}\text { Sig. } \\
\text { Sig. }\end{array}\end{array}$ & $\begin{array}{r}5 \\
61,5 \\
28 \\
00\end{array}$ & \\
\hline
\end{tabular}

El análisis dio a conocer cuántos y cuáles son realmente los factores del capital estructural (Tabla $\mathrm{N}^{\circ} 2$ ). El primer factor incluye indicadores referidos a la innovación, la comunicación y el acceso a la información, cuyo porcentaje de varianza explicada es 35,153 . El segundo factor se refiere al uso de técnicas informáticas, la gestión de la empresa y la preocupación por la calidad, que indican el modo de ser y trabajar de la empresa, con un porcentaje de varianza explicada de 26,663 y que sumadas hacen $61,816 \%$, superando el mínimo exigido del $50 \%$.

Tabla $\mathbf{N}^{\circ} 3$

AFE del Capital Relacional

\begin{tabular}{|c|c|c|}
\hline \multirow{2}{*}{ indicadores de Escala } & \multicolumn{2}{|c|}{ Factor } \\
\hline & 1 & 2 \\
\hline $\begin{array}{l}\text { CR6: Gestón de informacon de proveedores } \\
\text { CR2: Calidad de atencion al cliente } \\
\text { CR5: Gestion de informacion de clientes } \\
\text { CR1: Relación cor el cliente } \\
\text { CR3: Conocimiento de necesidades del cliente } \\
\text { CR4: Comunicación por internetcan el clente } \\
\text { CR7: Desarrollo de alianzas y convenios }\end{array}$ & $\begin{array}{l}.905 \\
.843 \\
.837 \\
.822 \\
.755\end{array}$ & $\begin{array}{l}898 \\
870\end{array}$ \\
\hline \%de varianza explicada & 49,720 & 23.060 \\
\hline \%o de varianza acumulada & 49,720 & 72,780 \\
\hline $\begin{array}{l}\text { Medida de adecuacion muestral de Kaiser-Meyer-Okin } \\
\text { Prueba de esfericidad de Chi-cuadrado aproximado } \\
\text { Bartett } \\
\qquad \begin{array}{l}\text { Sig. } \\
\text { Sig. }\end{array}\end{array}$ & $\begin{array}{r}68 \\
68.2 \\
2 \\
.00\end{array}$ & \\
\hline
\end{tabular}

El análisis dio a conocer cuántos y cuáles son realmente los factores del capital relacional (Tabla $\mathrm{N}^{\circ} 3$ ). El primer factor está referido a la relación de la empresa con sus proveedores, el conocimiento, la calidad y relación con sus clientes, con un porcentaje de varianza explicada es de 49,720.El segundo factor se refiere a la comunicación con sus clientes y a la fidelidad de las alianzas y convenios de la empresa que el reconocimiento en el mercado de sus productos y/o servicios; siendo su varianza explicada de 23,060 y que sumadas alcanzan un $72,780 \%$, superando el mínimo exigido del $50 \%$.

Comprobada la magnitud y significancia de las cargas factoriales se procede a la verificación de la hipótesis expresada en el análisis de regresión. 
Tabla No 4

AFE Relación Causal del Capital Intelectual y la Competitividad

\begin{tabular}{|c|c|c|c|c|c|c|}
\hline \multirow[t]{2}{*}{ Modela } & \multicolumn{2}{|c|}{$\begin{array}{l}\text { Coeficientes no } \\
\text { estandarizados }\end{array}$} & \multirow{2}{*}{$\begin{array}{l}\begin{array}{c}\text { Coefcientes } \\
\text { fipificados }\end{array} \\
\text { Beta }\end{array}$} & \multirow[b]{2}{*}{$t$} & \multirow[b]{2}{*}{ Sig. } & \\
\hline & B & Error tio. & & & & $\mathrm{R}^{2}$ \\
\hline (Constante) & $-1,491$ & 5,878 & & .254 & 803 &, 679 \\
\hline Capital Intelectual & .450 & .077 & .824 & 5,822 & 000 & \\
\hline
\end{tabular}

El resultado del análisis fue la verificación de la hipótesis; tal como se muestra en la Tabla № 4 anterior y en la figura siguiente (Figura $\mathrm{N}^{\circ} 2$ ); confirmando la mejor contrastación con un coeficiente estandarizado Beta $=0,824$ y un estadístico $t=5,822$ significativamente diferente de cero; indicando a la vez que el conjunto de los activos del capital intelectual, ejercen una influencia positiva mucho mayor ( $82.40 \%$ ) en la competitividad de las empresas que alguno de sus componentes en particular. En cuanto a $\mathrm{R}^{2}$ (Tabla $\mathrm{N}^{\circ} 4$ ) muestra un resultado satisfactorio ya que el poder explicativo del modelo completo es de $67.90 \%$ superior a los resultados parciales de los componentes del capital intelectual, indicando una vez más que se debe considerar al capital intelectual en su conjunto.

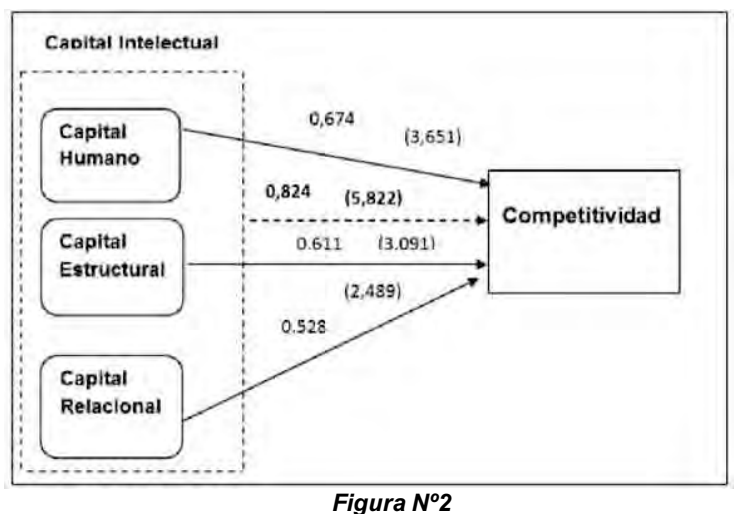

Resumen de Contrastación de Hipótesis

\section{Discusión}

El análisis de la realidad y la revisión de literatura inspiraron el problema de investigación. Éste se basa en la constatación de que el mundo empresarial cambió debiendo, por tanto, los empresarios e investigadores encontrar factores "productivos" determinantes de la ventaja competitiva sostenible adecuada a la realidad empresarial actual. En este contexto, se hace esencial encontrar los factores basados en el conocimiento capaces para crear ventajas y riqueza para las empresas.
Según el mundo actual y real de los negocios, las empresas que están en condiciones de exportar, han de cumplir con el volumen solicitado por el importador, calidad del producto requerida en el mercado de destino, ofrecer un precio competitivo y una utilidad razonable, clara garantía en el tiempo de entrega y servicio post-venta requerido.

Para el logro de los requisitos anteriores (Competitividad), la empresa debe estar fortalecida en el mercado nacional, con una estructura orgánica sólida, con recursos tecnológicos, financieros y humanos eficientes (Capital intelectual) que logren cumplir los objetivos estipulados.

Los estudios científicos desarrollados en esta problemática no precisan cómo identificar, definir o combinar los activos del capital intelectual. Es así, que se ha desarrollado el modelo propuesto de medición, basado en la verificación de que si el capital intelectual (por acción individual 0 conjunta de sus componentes) influye positivamente en la Competitividad de las empresas de este sector.

\section{Referencias bibliográficas}

1. Aguilar, I. (1999) Competitividad, Flexibilidad y rotación de personal en la industria maquiladora del televisor. COLEF. México.

2. Anderson, Tatham y Black. (1999). Analisis multivariante. Prentice Hall Iberia. Madrid.

3. Brooking, A. (1997). El Capital Intelectual. El Principal Activo de las Empresas del Tercer Milenio, Paidós Ibérica S. A., Barcelona.

4. Bueno, E. (1998).El Capital Intangible como Clave Estratégica en la Competencia Actual. Boletín de Estudios Económicos, vol. 53.

5. Carmeli, A., y Tishler, A. (2004). The Relationships between Intangible Organizational Elements and Organizational Performance, Strategic Management Journal, vol. 25.

6. Dierickx, I. y Cool, K. (1989). Asset Stock Accumulation and Sustainability 
of Competitive Advantage.Management Science, vol. 35, pp. 1504-1513.

7. Euroforum Escorial (1998). Medición del Capital Intelectual. Modelo Intelect, I.U. Madrid.

8. Ordoñez de Pablos, P. (2004). Las cuentas de capital intelectual como complemento del informe anual. Economía Industrial. ISSN 04222784 , № 357.

9. Pavez, A.(2000).Modelo de implantación de Gestión del Conocimiento y Tecnologías de Información para la Generación de Ventajas Competitivas. Valparaíso, Chile

10. Pelayo, C. (2008). La competitividad.

11. Porter Michael. (2003) Ventaja Competitiva. Creación y Sostenimiento de un Desempeño Superior. Ed. CECSA. México.

12. Stewart, T. A. (1998). La nueva riqueza de las organizaciones: el Capital Intelectual, Ediciones Granica, Barcelona.

13. Valdes Luigi.(2002) La revolución empresarial del siglo XX. Conocimiento y capital intelectual: Las nuevas ventajas competitivas de la empresa. Ed. Norma Bogotá.

14. Villareal Rene et al. (2003) La empresa competitiva sustentable en la era del capital intelectual. Ed. McGraw Hill.

Recibido: 28/09/13

Aceptado para publicación:

$15 / 11 / 2013$ 